\title{
Jugements de valeur en santé publique
}

\section{Jean Martin}

Dr méd., membre de la rédaction

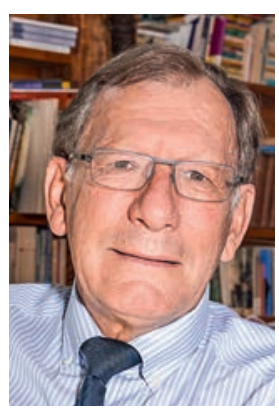

Référence

1 Finkel AM. A Guided Tour through Inevitable Value Judgments. A Hastings Center Spe cial Report, «Governance of Emerging Technologies», 2018, p. $30-45$.
En médecine et santé publique, les problèmes sont de plus en plus complexes, multifactoriels, demandant des appréciations en conséquence, à savoir multicritères. J'ai été intéressé par un article de Adam M. Finkel [1], qui a été aux Etats-Unis le patron du domaine Régulation de la Occupational Safety and Health Administration, conseillant le Gouvernement sur les mesures à prendre - ou ne pas prendre - pour protéger la santé au travail (domaine où des analyses coût-bénéfice jouent un rôle important). Parmi les questions qui se posent:

- Les dangers anthropogéniques sont-ils des cibles plus importantes pour la régulation que les dangers naturels?

- La société devrait-elle réguler les actes du registre de la commission, de l'omission, ou les deux?

- Devons-nous envisager des régulations pertinentes, mais qui peuvent sembler futiles au vu de l'importance du problème ("goutte d'eau dans la mer»)?

- Le bénéfice sociétal net est-il toujours la bonne mesure, même si pour beaucoup de personnes le bénéfice individuel diminue?

- Cherchons-nous un résultat bon, meilleur qu'avant, ou le mieux qu'on puisse atteindre? (Question du mieux qui peut être l'ennemi du bien...)

- Quelle définition d'optimal appliquons-nous parmi de nombreuses définitions?

- Cherchons-nous à améliorer le bien-être ressenti par les bénéficiaires ou un mieux-être objectif?

- Considérons-nous comme bénéfiques la réduction d'atteintes à l'environnement naturel même si la santé et le bien-être humains ne sont eux pas affectés? (Vers des droits de la nature?)

- Sur quel laps de temps (court, moyen ou long terme) nos analyses doivent-elles porter?

- Incluons-nous ou ignorons-nous les effets des dangers et de leur contrôle hors de notre pays?

- Devons-nous inclure les vues privées des citoyens dans l'appréciation de la valeur d'une régulation?

- Les analystes doivent-ils exclure les bénéfices (et les coûts) «non quantifiables»?

- Devons-nous envisager des actions qui transfèrent du bien-être au sein d'une population, entre différentes parties, mais n'entrainent pas de bénéfice net global?
- Les valeurs en question doivent-elles exclure les considérations d'altruisme?

- Devons-nous, s'agissant de maladies ou accidents fatals, considérer les vies perdues ou les années de vie perdues?

- Toutes les années de vie doivent-elles être considérées de manière équivalente ou faut-il utiliser la notion d'«années de vie ajustées pour la qualité»?

- La longévité est vue come un bénéfice privé, mais doit-on en tenir compte comme un coût social?

- Qui est qualifié en tant que "partie prenante» (notion de stakeholder)?

- Certaines parties prenantes ont-elles, le cas échéant, des possibilités particulières (extra opportunities) de participer aux réflexions et orientations?

- Les analystes devraient-ils surtout éviter de donner une impression erronée de confiance dans leurs résultats, ou doivent-ils éviter d'entourer leurs estimations de "Il reste beaucoup d'incertain»?

- Toutes les sources d'incertitude doivent-elles être mentionnées, notamment les théories scientifiques plausibles mais mutuellement incompatibles?

- Comment les ressources doivent-elles être distribuées entre la prévention et la compensation après coup? (Les sociétés - trop - libérales tendent à attendre de constater les dégâts avant d'agir - J. M.)

- Est-ce à l'autorité publique d'apporter la preuve qu'un acte est / a été volontaire, ou est-ce à celui qui a violé la règle de prouver que ses actions n'étaient pas volontaires?

Une carrière de médecin officiel/cantonal m'a sensibilisé à ces interrogations, pertinentes aussi au modeste niveau d'un canton. Réguler oui ou non, combien, comment? L'enjeu est, compte tenu des faits, de trouver le juste équilibre entre les intérêts en présence.

Un conseiller d'Etat sous lequel j'ai œuvré disait: «Un Etat bon à tout est un Etat bon à rien.» L'autorité ne doit pas se mêler de tout, les individus et les groupes doivent rester libres d'entreprendre. Cela étant, les pouvoirs publics ont mission de promouvoir le bien commun, qui dans notre esprit ne passe pas souvent avant les intérêts individuels! Maintenir une dimension de valeurs et de buts partagés est plus que jamais nécessaire. 